\title{
Gestación gemelar con muerte intraútero de uno de los fetos en un hospital general de Perú
}

\author{
Liliana Luna-Capcha, ${ }^{1, c}$, Hernán Adán Montalvo-Uvidia,c, Hebert Omar Palomino-Donayre ${ }^{1, c}$, Ivette Vasquez-Cucho ${ }^{1,2, b}$, Jorge Ybaseta- \\ Medina, ${ }^{1,2, a}$ \\ Facultad de Medicina, Universidad Nacional San Luis Gonzaga. Ica, Perú ${ }^{1}$ \\ Hospital Santa María del Socorro. Ica, Perú2 \\ Médico especialista en ginecología y obstetriciaa , residente de la especialidad de ginecología y obstetricia ${ }^{b}$, estudiante de medicinac
}

\begin{abstract}
$E^{\prime}$ embarazo múltiple está asociado a un aumento en la mortalidad y morbilidad perinatal, comparado con el embarazo único. Una complicación grave que afecta al embarazo gemelar, es la muerte fetal in útero de un gemelo, situación que implica un riesgo de morbilidad/mortalidad para el feto sobreviviente y la embarazada. Presentamos un caso de una paciente de 29 años que recibió tratamiento de infertilidad de baja complejidad, resultando una gestación gemelar monocoriónico diamniótico, que nació a las 38 semanas de gestación presentando un feto muerto desde las 21 semanas, fue sometida a cesárea por inicio de trabajo de parto y feto de situación transversa, evolucionando favorablemente.
\end{abstract}

Palabras claves: embarazo gemelar, óbito fetal, hipofibrinogenemia (fuente: DeCS BIREME).

Autor Corresponsal: Jorge Ybaseta Medina. Dirección: Calle Fermin Tanguis J-152. Ica, Perú. Teléfono: (51) 956747971. Correo electrónico: jorgeybaseta@yahoo.es

\section{INTRODUCCIÓN}

Los embarazos múltiples son frecuentes y su incidencia está aumentando debido al uso de las técnicas de reproducción asistida $(1,2)$ el Hospital Santa María del Socorro de la provincia de Ica, no escapa de esta realidad, de 10000 embarazos que han registrado en el sistema informático perinatal, el 0,3\% son embarazos gemelares (31) y de estos se presentó un embarazo gemelar con óbito.

Los embarazos múltiples se asocian con un porcentaje más alto de complicaciones, con tasas más altas de mortalidad, malformaciones, anomalías del desarrollo y bajo peso al nacer (2). Uno de sus complicaciones es la muerte de un gemelo in útero, descrito hasta en el 6,2\% de los gemelos (3).

En el embarazo gemelar se comprenden dos fenómenos muy diferentes, esto no solo tiene importancia teórica, sino también clínica y práctica. En primer lugar, dos ovocitos pueden ser fecundados por dos espermios diferentes: gemelos dicigóticos. En segundo lugar, un cigote ya en vías de blastogénesis puede engendrar dos embriones separados: gemelos monocigóticos o también "gemelos idénticos". Tienen la misma constitución genética y son siempre del mismo sexo (4).

Los gemelos monocigoticos tienen un origen ontogénico posterior a los dicigóticos, se originan en el desarrollo embrionario. Se pueden distinguir las siguientes posibilidades: 1) La partición en dos embriones se verifica en estado de mórula, dando lugar a dos huevos distintos, que simulan a los embarazos dicigoticos y como ellos son dicoriales; sin embargo, su sexo siempre será el mismo. 2) La partición se verifica en fase de blastocisto. Se formará una placenta única: monocorial, pero tendrá dos amnios: diamniotico. 3) La partición se verifica en el disco embrionario, dando lugar a gemelos con una sola placenta y un solo amnios o monoamnioticos. 4) Finalmente la duplicación tiene lugar en la fase de la embriogénesis, dando lugar a los monstruos dobles (4).

La nutrición en los embarazos monocigoticos suele estar más comprometida que en los dicigoticos, ya que los primeros es más frecuente que los dos fetos tengan que nu-

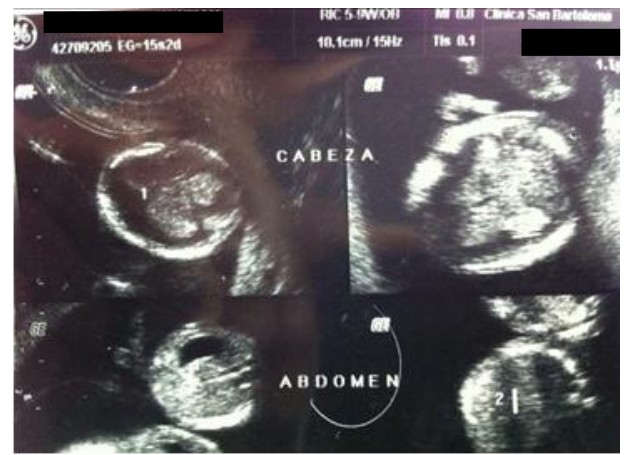

Figura 1. Gemelos discordantes.

trirse de una misma placenta. Cuando la placenta es una, o son dos, pero íntimamente soldadas (la existencia de una sola placenta, sea primitiva o secundaria) puede dar lugar a la anastomosis de los vasos placentarios con lo que se daría el espacio a una tercera circulación. Esta anastomosis pueden ser de tres tipos: A) Anastomosis venosa ( $5 \%)$. B) Anastomosis arterial (66\%). C) Anastomosis a la vez arterial y venosa $(29 \%)(4,5)$.

Esta disposición puede, aunque no siempre lo hace, colocar a los fetos en parabiosis. Esto puede generar que un feto se haga parasito del otro y se retrase en el desarrollo. Y esto da lugar a dos fenómenos: que el feto retrasado no desarrolle su corazón ni su sistema nervioso: holocardius amorphus o llamado simplemente "acardio". O bien que el feto retrasado muera y quede excluido del desarrollo intrauterino, pudiendo llegar a momificarse o a ser aplastado, dando lugar a un feto papiráceo. Otras veces la desaparición del gemelo es más precoz y no deja rastros. Es lo que modernamente 


\section{REPORTE DE CASO}

Paciente primigesta de 29 años de edad, ingresa al Hospital Santa María del Socorro de Ica por presentar dolor tipo contracción uterina, llega con el diagnóstico ecográfico de embarazo gemelar de 38 semanas, con el primer gemelo vivo en situación transversa y el segundo obitado desde la semana 21. Al examen ecográfico hay signos de una placenta tipo monocorial diamniótica. La gestante tiene antecedentes de tratamiento de infertilidad de baja complejidad, 06 controles prenatales y 03 ecografías observándose fetos discordantes (Figura 1), presentando el feto de posterior óbito un higroma a las 15 semanas de gestación (Figura 2).

Ai examen: Paciente en buen estado general, lúcida, orientada en tiempo, espacio y persona. Signos vitales dentro de los valores normales. Abdomen globuloso, con una altura uterina de $32 \mathrm{~cm}$, primer feto en situación transversa con frecuencia cardiaca de 136 latidos por minuto y el segundo feto obitado.

Dinámica uterina: 3 contracciones cada 10 minutos. (++), movimientos fetales $(++)$, tacto vaginal: incorporación de $70 \%$, dilatación en $0 \mathrm{~cm}$ y altura de presentación en 4.

La paciente fue internada con los siguientes diagnósticos: primigesta con tiempo de embarazo de 38 semanas y 6 días según fecha de última regla; embarazo gemelar con un feto obitado; feto vivo en situación transversa y preparto.

A tres horas de su ingreso, se evalúa a la gestante encontrándose: al tacto vaginal una dilatación de $2 \mathrm{~cm}$, incorporación $85 \%$, altura de presentación fetal de -4 . Se realizaron exámenes de biometría hemática y perfil de coagulación con resultados normales.

Al encontrarse el feto en situación transversa, se interviene quirúrgicamente a la paciente para un parto por cesárea, extrayéndose un feto papiráceo de longitud cráneo nalga de $20 \mathrm{~cm}$, diámetro biparental de $2 \mathrm{~cm}$; $y$ un feto vivo de sexo femenino con un peso de $3,8 \mathrm{Kg}$., talla de $48 \mathrm{~cm}$, perímetro cefalico de $37 \mathrm{~cm}$ y un puntaje de Apgar de 9 tanto en el primer como en el quinto minuto, evidenciando que cada uno de ellos tenía su propia bolsa amniótica (Figura 3). Placenta única de $19 \times 18 \times 2.5 \mathrm{~cm}$ y peso de 600 gr. No se presentaron complicaciones durante el procedimiento.

En el post operatorio evoluciona favorablemente. Se indican analgésicos, antibióticos. Se otorga el alta al cuarto día del puerperio, el hijo recibe lactancia materna sin ninguna dificultad.

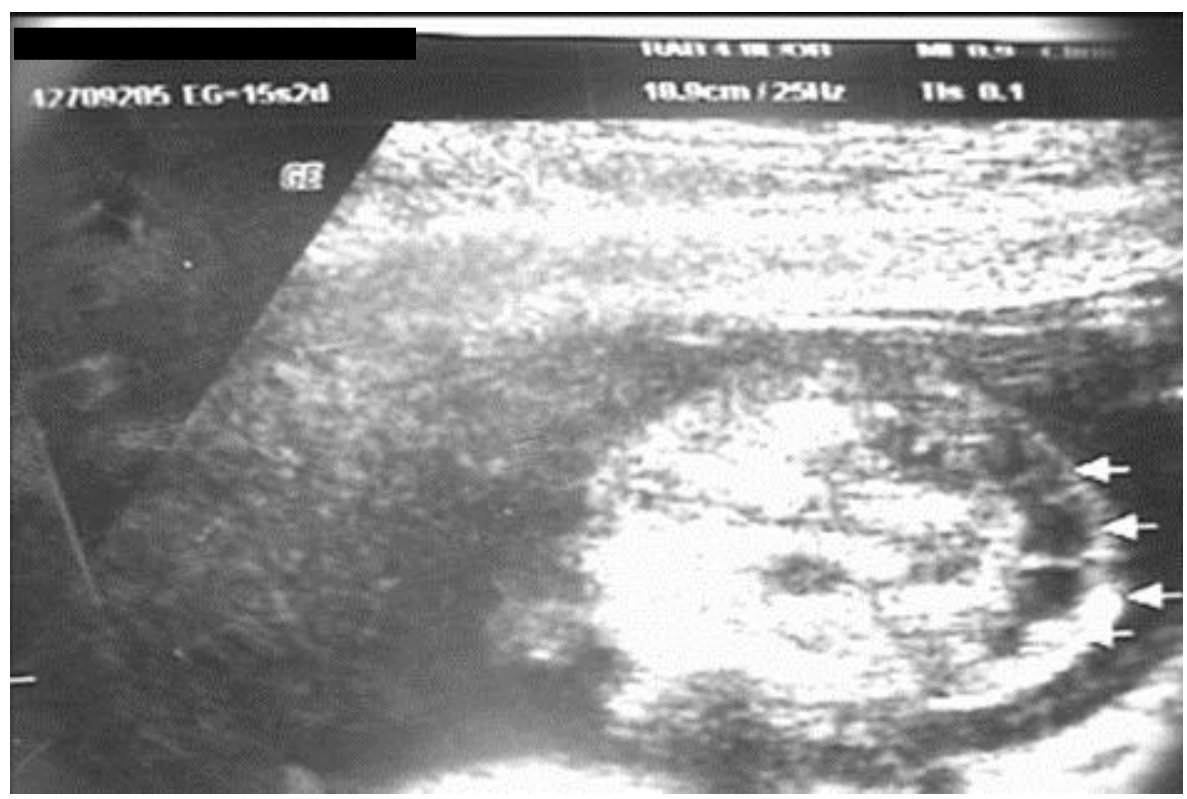

Figura 2. Higroma

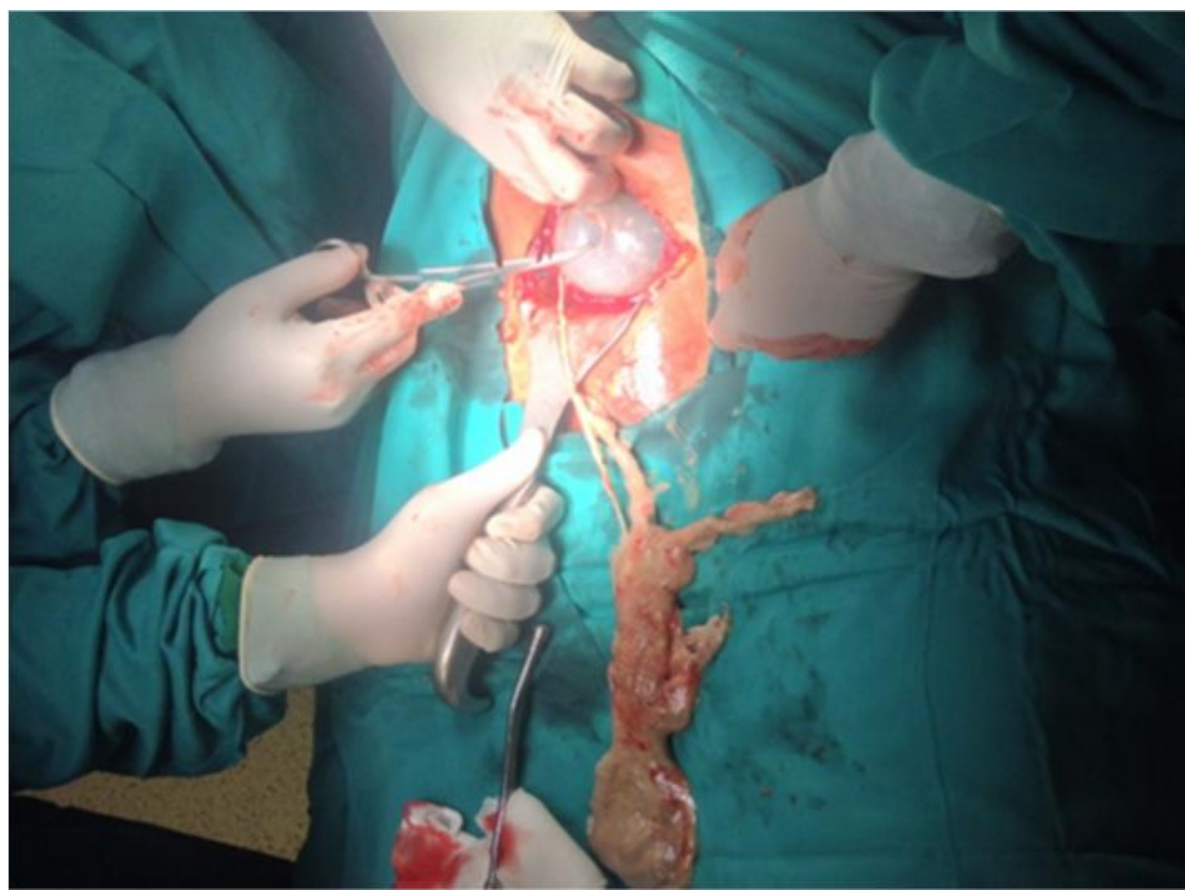

Figura 3. Feto papiraceo y segunda bolsa amniotica.

\section{DISCUSIÓN}

Los gemelos de placentas monocorionicas tiene una mortalidad mucho más elevada (2). Siendo esta tasa de mortalidad $25 \%$ a comparación de los dicorionicos 8,9\% (6).

Los gemelos monocorionicos diamnioticos tienen mayores posibilidades de sufrir trastornos del crecimiento, por el gradiente de circulación compartida, causal de mortalidad (7) como también malformaciones (2), congruentes con nuestro caso donde a las 15 semanas por hallazgo ecográfico se encuentra un higroma y se evidencia discordancia en crecimiento fetal (Figura 1 y Figura 2).
El término feto papiráceo se utiliza para describir un feto momificado asociado a una gestación gemelar o múltiple. La incidencia más alta es en gestaciones gemelares monocoriónicas. Su incidencia es baja, 1/12 000 nacidos vivos (8), congruente con los casos que se registran en nuestro hospital.

Según literatura revisada no se ha encontrado relación de este hallazgo con número de gestas, paridad o edad materna, aunque si se ha visto este fenómeno con mayor incidencia en embarazos gemelares monocigóticos en relación con los dicigóticos (9).

Cuando un gemelo muerto permanece in 
útero, realizaron con mayor frecuencia la evaluación prenatal del gemelo vivo a través de ultrasonografía, revisando corion, crecimiento fetal, el volumen de líquido amniótico, riñones, anatomía del sistema nervioso central, velocimetria doppler del cordón umbilical y perfil de coagulación tal como se describen en diversas literaturas que reportan casos similares.

\section{CONCLUSION}

El pronóstico es mejor para la madre y el recién nacido cuando el feto papiráceo aparece en un embarazo diamniòtico.

\section{REFERENCIAS BIBLIOGRÁFICAS}

1. Murphy KW. Intrauterine death in a twin: implications for the survivor. In: Ward $\mathrm{RH}$, Whittle $M$, editors. Multiple pregnancy. London: RCOG Press;1995:218-30.

2. Reece EA, Hobbins JC. Obstetricia Clinica/ Clinical Obstetrics. $3^{\mathrm{a}}$ ed. Buenos Aires: Editorial Medica Panamericana; 2010. Pag 48

3. Lee YM, Wylie BJ, Simpson LL, D'Alton ME. Twin chorionicity and the risk of stillbirth. Obstet Gynecol 2008;111(2 Pt 1):301-8.

4. Botella J y Clavero J. Tratado de Ginecología: Fisiología, Obstetricia, Perinatología, Ginecología y Reproducción. $14^{a}$ ed. Madrid: Díaz de Santos S.A.;1993. Pag 526, 527, 528.

5. Avery G, Fletcher M y Mac Donald M. Neonatología. Fisiología y manejo del recién nacido. 5 ta ed. Madrid: Medica Panamericana S.A.;2001. Pag. 137, 138.

6. Gomella, Cunningham, Eyal, Zenk. Neonatología: Manejo básico, Procedimientos, Problemas en la guardia, Enfermedades y Fármacos $.5^{\text {a }}$ ed. Buenos Aires: Editorial Medica Panamericana; 2009.Pag 533-

7. Pacheco J. Ginecología, obstetricia y reproducción. Vol 2. $2^{\mathrm{a}}$ ed. Tomo II. Lima: Revistas Especializadas Peruanas SAC; 2010. Pag 13064.

8. Vargas HV, Lujan IJ, Ibarra GA. Feto papiráceo complicación de un embarazo gemelar. Rev Hosp Jua Mex 2005; 72(4): 165-7.

9. Lujan IJ, Ibarra GA, Inclán BJ, Rascón AA, López CG. Feto papiráceo: Complicación de un embarazo gemelar. Clin Hosp Infant Edo Son 2005; 22(2): 145-7.

10. Moise K, Johnson A. Management of twintwin transfusion syndrome. UpToDate 19.1.2011. [Link]

11. O'Donoghue $K$, Rutherford $M$, Engineer $N$, Wimalasundera R, Cowan F, Fisk N. Transfusional fetal complications after single intrauterine death in monochorionic multiple pregnancy. BJOG 2009;116:804-12.

12. Mendoza LE, Sánchez RM, Camacho AA, Balcázar R, Rodríguez Al, Casian GA. Feto

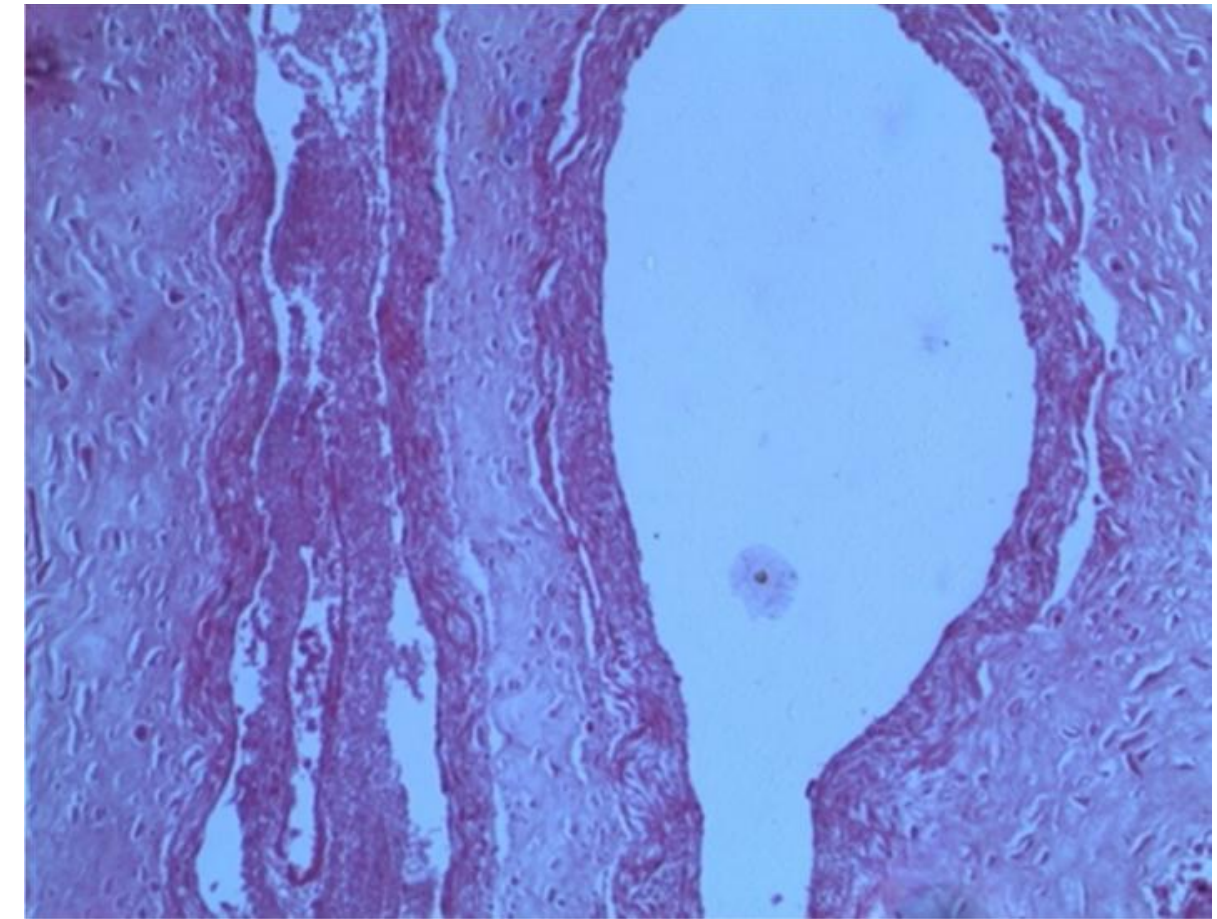

Figura 4. Vasos del cordon umbilical con signos de necrosis.

papiráce. Rev Hosp Jua Mex 2010; 77(2): 138140

13. García A, Iriarte R, Ríos V, Cortez M. Manejo del embarazo múltiple con óbito del segundo gemelo. Caso Clínico. UpToDate 19.09.2014. [Link]

14. Barba S. Carvajal J. Pautas de manejo del embarazo múltiple complicado con la muerte fetal in útero de un gemelo. Rev chil obstet ginecol 2012; 77(2): 159 - 168 .

15. Fernández-Miranda $M$, Cruceyra $M$, Rodríguez-González R, Magdaleno-Dans F, Omeñaca F,González A. Gestación gemelar con muerte intraútero de uno de los fetos: pronóstico materno y neonatal del feto superviviente. Ginecol Obstet Mex 2011;2012;80(4):254-262 
FINANCIAMIENTO: Autofinanciado.

CONTRIBUCIONES DE AUTORÍA: LLC, HAMU, HOPD, IVC y JYM participaron en la concepcion, recoleccion, redaccion y revisión crítica del artículo y en la aprobación de su versión final del presente reporte.

\section{Twin pregnancy with intrauterine death of one fetus in a general hospital in Peru}

U ultiple pregnancy is associated with increased perinatal mortality and morbidity, compared with singleton pregnancy. A serious complication that affects twin pregnancy is fetal death in utero of one twin, a situation that involves risk of morbidity / mortality for the surviving fetus and pregnant. We present a case of a patient of 29 years who received infertility treatment low complexity, resulting diamniótico monochorionic twin pregnancy, born at 38 weeks of gestation presenting a standstill from 21 weeks fetus underwent cesarean start working delivery and fetal transverse lie, evolving favorably.

Key words: twin pregnancy, stillbirth, hypofibrinogenemia (source: MeSH NLM)

\section{CITA SUGERIDA.}

Luna-Capcha L, Montalvo-Uvidia HA, Palomino-Donayre HO, Vasquez-Cucho I, Ybaseta-Medina J. Gestación gemelar con muerte intraútero de uno de los fetos en un hospital general de Perú. Rev méd panacea.2014; 4 (1):22-25. 